\title{
THE CRITICAL ROLE OF BONE MARROW STUDY AND IMMUNOPHENOTYPING IN THE EVALUATION OF ANAEMIA OF UNDETERMINED ETIOLOGY; CASE PRESENTATION AND REVIEW OF LITERATURE
}

${ }^{1}$ Chukwudi O. Okani, ${ }^{2}$ John Aneke, 3Prince Ele, ${ }^{4}$ Uchenna Eke

'Department of Histopathology, Chukwuemeka Odumegwu Ojukwu University, Awka Campus, Anambra State ${ }^{2}$ Department of Haematology and Blood Transfusion, Nnamdi Azikiwe University, Nnewi Campus, Anambra State ${ }^{3}$ Department of Medicine, Nnamdi Azikiwe University, Nnewi Campus, Anambra State ${ }^{4}$ Grace and Truth Specialist Hospital, Nnewi, Anambra State

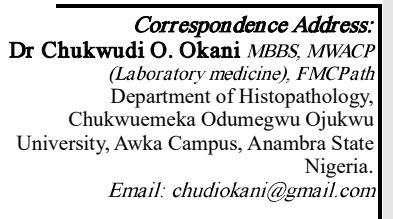

\section{ABSTRACT}

Marrow infiltration by cancer is a known cause of anaemia, and the diagnosis and management are often without difficulty. However, in some cases, the primary source of malignancy may be elusive with unexplained anaemia as the most important presenting complaint. We report a case of a 71-year old male who presented to hospital with shortness of breath, generalized body weakness, poor appetite, marked weight loss and low haematocrit (of $0.20 \mathrm{~L} / \mathrm{L}$ ), low corrected reticulocyte count $(0.4 \%)$, mild reduction in all cell lines (with normal morphology) as well as mildly hypochromic, microcytic red cells on peripheral blood film examination. Bone marrow studies revealed infiltration by malignant carcinoma. This finding led to the diagnosis of marrow infiltration by carcinoma; histology and immunophenotyping subsequently confirmed the prostate as the primary site. A definitive diagnosis of myelophthisic anaemia secondary to prostate carcinoma was thus made and patient was consequently referred to the urology team for further management.

KEYWORDS: Bone marrow, myelophthisis, trephine biopsy, immunohistochemistry

\section{INTRODUCTION}

$\mathrm{P}$ atients with marrow infiltration by cancer may initially present with anaemia even in the absence of overt primary neoplastic lesion. Clinical evaluations with associated laboratory investigation which include bone marrow studies are often important in making an accurate diagnosis and instituting the proper management plan. Detection of metastatic neoplastic marrow infiltration is essential in staging of tumuor. This finding definitely will impact on the clinical course of the disease, choice of chemotherapeutic agent, overall survival, and appropriate dosage titration to avert further marrow suppression. One test with invaluable benefit in bone marrow studies is immunophenotyping.

\section{CASE REPORT:}

We report a case of a 71-year old male Nigerian who presented to our hospital with a year history of shortness of breath, generalized body weakness, poor appetite and marked weight loss. There was absence of vomiting, diarrhea, constipation, fever, jaundice, cough, dysuria or features suggestive of bladder outflow obstruction. His hemoglobin genotype was AA and he had not been transfused with blood in the past. In addition, he was not a known diabetic, hypertensive, alcoholic or tobacco user and no bleeding diathesis in the remote or recent past. Physical examination revealed an elderly man with tachycardia (pulse rate of 132/minute) in respiratory distress, with moderate to severe mucosal pallor. There was no conjuctival icterus, pedal oedema or peripheral lymph node enlargement. Also of note was the absence of significant clinical findings in the abdominal, chest and central nervous system examinations.

Laboratory work up was significant for moderate-tosevere anaemia (haematocrit of $0.20 \mathrm{~L} / \mathrm{L}$ ), low corrected reticulocyte count $(0.4 \%)$, mild reduction in all cell lines (with normal morphology) as well as mildly hypochromic, microcytic red cells on peripheral blood film examination. Results of stool microscopy, stool for occult blood, urinalysis, abdominal ultrasound scan, fasting lipid profile, retroviral screening, renal and liver function tests were not remarkable.

A clinical diagnosis of anaemia in an elderly man, most probably due to marrow underproduction was therefore made. Due to the fact that the cause of anaemia was not apparent from history, examination and preliminary investigation findings, further work up in the form of bone marrow studies were recommended. Bone marrow aspiration showed focal infiltration by foreign cells with mild to moderate reduction in the myeloid and megakaryocytic elements. Trephine histology revealed sheets of foreign spindle cells admixed with some polygonal forms, with foci of necrosis and active residual marrow. A provisional histologic diagnosis of atypical 
infiltration of the bone marrow was made with emphasise on the possibility of stromal or poorly differentiated metastatic carcinoma. It is of note to consider the pleomorphic cytologic appearances of cells in the bone marrow. Some cells in some histologic foci appear spindly while some are polygonal in shape. This dimorphic cellular architecture makes a diagnosis of spindle cell or carcinomatous infiltration particularly difficult. Figures 1 to 2 show different histologic patterns of the tumour cells in the bone marrow.

This case was further subjected to Immunohistochemistry analysis by staining the bone marrow tissue with a panel of antibodies which include: AE1/AE3, Desmin and Vimentin. Special stains revealed strong immunoreactivity with cytokeratins (AE1/AE3) in the area containing atypical infiltration (Figure 3A), and negative for Desmin. The non-neoplastic stromal elements show Desmin positivity as internal control (Figure 3B). There was focal nonspecific immunostaining with vimentin in the necrotic area (Figure 3C). We however, concluded our final histologic diagnosis as a metastatic carcinoma to the bone marrow.

The patient was further investigated for the source of occult epithelial malignancy. A transurethral ultrasound of the prostate performed showed prostatic enlargement with coarse heterogeneous echo texture, ill defined outlines and incomplete capsule. These findings were suggestive of carcinoma of the prostate. The histology of the trucut prostatic biopsy revealed an adenocarcinoma of the prostate with Gleason grade 4, score 8 . The patient is being co-managed with the urology team as a case of myelophthisic anaemia secondary to carcinoma of the prostate.

\section{DISCUSSION}

Metastatic cancers of the bone marrow have been reported in all types of malignancies. Carcinomas arising in the prostate, breast, lung and neuroblastoma have been noted as common primary sites. Prompt diagnosis is necessary for tumour staging, prognostication and choice of chemotherapy. Histological assessment of biopsy specimens is the sine qua none in detection of residual disease. 1 Consequently, bone marrow infiltrates are diagnosed by bone marrow aspirates and trephine biopsies and these two techniques are known to be sensitive. However, the sensitivity of the trephine biopsy is higher than that of the bone marrow aspiration. Taking bilateral biopsies or getting a single large biopsy is known to improve the sensitivity; it is therefore, of importance to regard these two procedures as complementary. 2 Moreover, it is not unusual to miss the diagnosis of marrow infiltration in smears of an aspirate even when the trephine biopsy is positive for metastasis. However, trephine biopsy may be normal in few occasions where tumuor cells are seen in aspirate smears.3, 4 In this index case, atypical tumuor cells were seen in both aspirate smears and in the trephine biopsy. A precise diagnosis requires a histological examination with the support of immunophenotyping and complemented by radiological investigations, molecular studies and electron microscopy in some situations.5 Some researchers from different parts of the world have reported metastatic carcinomas to the marrow with primary sites from small carcinoma of lung and prostate carcinoma, 6, 7 infiltrating lobular carcinoma of breast.

The hematological findings seen in patients with marrow infiltration are heterogeneous. Some present with pancytopenia while some present with anemia, thrombocytopenia and/ or leucopenia.8 And, in this index case, the patient presented with normochromic hypochromic anemia, leukopenia and thrombocytopenia. The profile of anemia was similar to the findings documented by other researchers.9 About a $30-50 \%$ of patients with marrow infiltration may present with leukoerythroblastic changes. Metastatic carcinomas infiltrating the marrow may be focal or diffuse. Collagen deposition with fibrosis is a common feature seen on bone marrow with metastatic deposits.10 -12 Fibrosis was apparent in this case being reported.

It is not uncommon to see undifferentiated tumour infiltrating the marrow; and this often pose diagnostic challenge. In such situations, an array of differential diagnoses must be borne in mind and these include: undifferentiated carcinomas, melanomas and NonHodgkins lymphoma. Immunophenotyping is almost inevitable in resolving the primary source of neoplasm.13, 14 Prompt and accurate diagnosis is of essence in bone marrow metastasis primarily originating from breast and prostate. This is because of their sensitivity to hormonal therapy. The patient in this index case benefited from the precise diagnosis as his condition was noted to have dramatically improved following the bilateral orchidectomy and administration of anti-androgenic agents.

Performing Immunohistochemistry on bone marrow metastatic deposits has helped in detection of primary tumour sources, and further phenotypic typing like the use of antibody panels for detection of hormone receptors as in the case of breast cancers with bone marrow metastasis is known to impact positively on 
prognostic outcome in patient management. Prostate specific antigen (PSA) also shows positivity in bone marrow metastases from prostate adenocarcinoma. 15 It is of interest to note that bone marrow is frequently involved by prostatic carcinoma even without evidence of skeletal disease. 16 In this study, the PSA of the patient was mildly elevated without any form of skeletal involvement.

\section{Conclusion:}

This case demonstrates the benefit of multidisciplinary approach in patient care in certain clinical conditions and highlights the need for high index of suspicion when diagnosis is problematic. The critical role of bone marrow studies and immunophenotyping in the evaluation of certain anaemias has been emphasized.
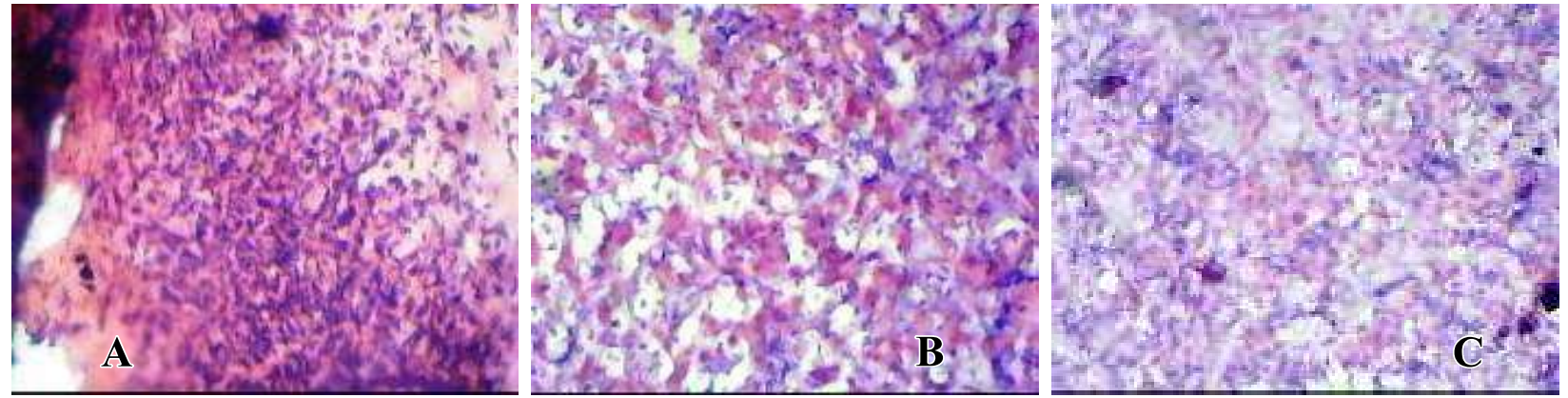

FIGURE 1. A, Low power micrograph showing infiltration of foreign spindle-shaped cells into the marrow. B, Showing focus of residual marrow elements. C,Area of necrosis with marrow elements admixed with some atypical cells.
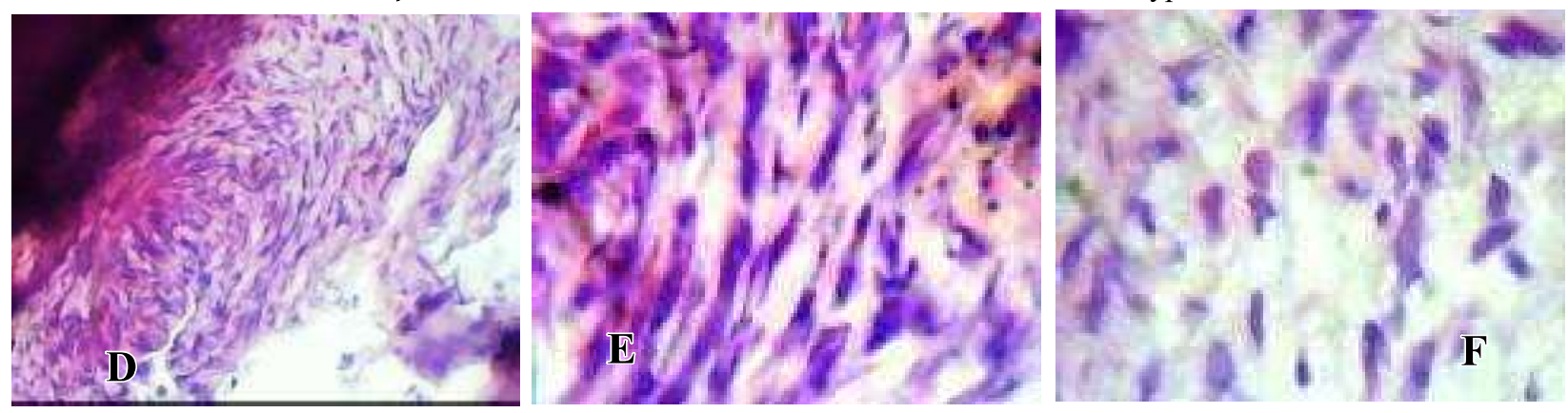

FIGURE 2. D, Low power micrograph showing spindle-shaped cells. E, Higher magnification showing spindle-shaped cells. F, Spindle cells admixed with some polygonal cells.
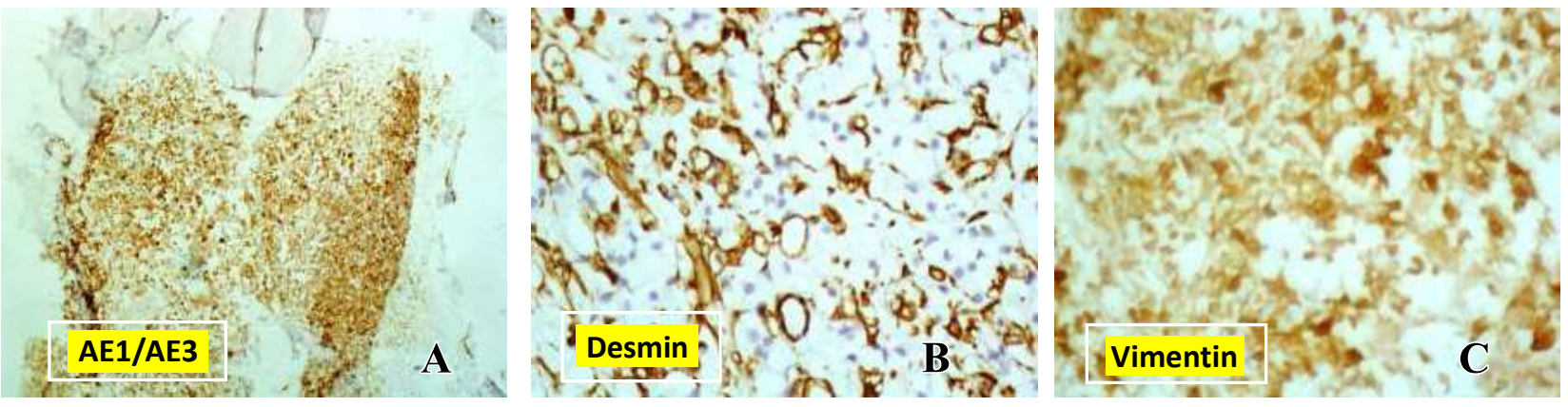

FIGURE 3. A, Spindle-shaped cells showing strong immunoreactivity to AE1/AE3, indicating epithelial origin despite being spindly. B, This focus shows stromal marrow elements with Desmin immunoreactivity while the background neoplastic cells are non-reactive. C, This shows a non-specific staining with Vimentin in foci containing necrotic tissue debris.

\section{References}

1. Zehentner BK. Detection of disseminated tumuor cells: strategies and diagnostic implications. Expert Rev Mol Diagn 2002; 2: 41-48.

2. Mohanty SK, Dash S. Bone marrow metastasis in solid tumuors. Indian J Pathol Microbiol 2003; 46: 613-616.
3. Singh G, Krause JR, Breitfeld V. Bone marrow examination for metastatic tumuor. Cancer 1977; 40:2317-2321.

4. Savage RA, Hoffman GC, Shaker K. Diagnostic problems involved in detection of metastatic neoplasms by bone marrow aspirate compared with needle biopsy. Am J Clin Pathol 1978; 70: 
623-627.

5. Mohanty SK, Dash S. Bone marrow metastasis in solid tumuors. Indian J Pathol Microbiol 2003; 46: 613-616.

6. Beena Brahmbhatt, Biren Parikh, Manoj Shah. Bone Marrow involvement by Metastatic solid Tumuors. GUJARAT MEDICAL JOURNAL / AUGUST-2014 Vol. 69 No. 2

7. Moid F, DePalma L. Comparison of relative value of bone marrow aspirates and bone marrow trephine biopsies in the diagnosis of solid tumuor metastasis and Hodgkin's lymphoma. Institutional experience and literature review. Arch Pathol Lab Med 2005; 129: 497-501.

8. Jamila, Khalid Hassan, Nadeem Ikram. Peripheral Blood Parameters in Patients of Non-Hodgkin's Lymphomas Showing Bone Marrow Infiltration. Journal of Islamabad Medical \& Dental College (JIMDC); 2012(2):85-88

9. Sar R, Aydogdu I, Ozen S, Sevinc A, Buyukberber $S$ Metastatic bone marrow tumours : a report of six cases and review of the literature. Haematologia 2001;31:215-223.
10. Rubins JR. The role of myelofibrosis in malignant myelosclerosis. Cancer 1983; 51:308-311.

11. Kiely JM, Silverstein MN. Metastatic carcinoma simulating agnogenic myeloid metaplasia. Cancer 1969; 24: 1041-1044.

12. Spector JI, Levine PH. Carcinomatous bone marrow invasion simulating acute myelofibrosis. Am J Med Sci 1973; 266: 145-148.

13. Gatter KC, Abdulaziz Z, Beverley P, Corvalan JR, Ford C, Lane EB, et al. Use of monoclonal antibodies for the histopathological diagnosis of human malignancy. J Clin Pathol 1982; 35: 12531267.

14. Gatter KC, Ralfkiaer E, Skinner J, Brown D, Heryet A, Pulford, et al. An immunohistochemical study of malignant melanoma and its differential diagnosis from other malignant tumuors. J Clin Pathol 1985; 38: 1353-1357.

15. Papac RJ. Bone marrow metastases - A review. Cancer 1994; 74: 2403-2413.

16. Rundles RW, Jonsson U. Metastases in bone marrow and myelophthisic anemia from carcinoma of the prostate. Am J Med Sci 1949, 218: $242-250$. 